\title{
The effect of friction force and cavitation on wear in piston-cylinder assemblies
}

\author{
Polychronis Dellis ${ }^{1 *}$ \\ ${ }^{1}$ School of Mechanical Engineering Educators, Assistant Professor - Scientific Associate, ASPETE, \\ Heraklion, Attiki, 14121, Athens
}

\begin{abstract}
Both in engines and test rigs, cavitation in piston-ring lubrication is a subject studied by many researchers in the past. Although there is no sufficient evidence of cavitation erosion on the surface of the liner, this phenomenon is studied as part of the lubricant transport process. During the transport process the lubricant enters the combustion chamber. The combustion products of consumed oil might contribute to exhaust gas emissions and with emission legislation becoming more stringent, it is important that oil consumption is reduced to the lowest level possible. The role of the piston-rings is becoming more complex with the imposed requirements for lower oil consumption and friction. The piston-ring pack role on engine performance, durability and wear, is becoming on the other hand, more demanding. Between the piston-ring and cylinder liner, cavitation occurs as a result of two-phase liquid flow. Cavitation has long been recognized to degrade performances in most engineering applications and its effect in piston-ring lubrication is that it alters the oil film pressure profile, generated at the converging-diverging wedge of the piston-ring. An area of the piston-ring surface is void, corresponds to subatmospheric pressures and thus, the piston-ring load capacity is altered. Two experimental rigs were used, a simplified single-ring test rig that simulates the piston-ring liner movement at speeds corresponding to idle but the movement is reversed and a single cylinder diesel engine that was used for visualization results only, after the necessary modifications.
\end{abstract}

\section{Introduction}

The formation of cavities and their subsequent disposition affects the pressure generated in the continuous thin lubricant film, and hence, any integrated quantities such as the load capacity of bearings. Understanding of the flow in a simplified test rig rather than in an ordinary ring pack provides information of practical importance and leads to characterisation of the lubricant transport phenomena in the piston-ring pack of production engines [1].

Cavitation has long been recognized to degrade performances in most engineering applications and its effect in piston-ring lubrication is that it alters the oil film pressure profile, generated at the converging-diverging wedge of the piston-ring and liner interface. An area of the piston-ring surface is void, corresponds to subatmospheric pressures and

\footnotetext{
${ }^{1}$ Corresponding Author, pasd@city.ac.uk
} 
thus, the piston-ring load capacity is altered. The load carrying capacity of the interface is the integral of the pressure distribution, the lubricant film thickness, the friction force and the lubricant flow rate [2].

A non shear-thinning mineral lubricant having viscosity equal to the lower Newtonian viscosity value of a shear thinning polymeric lubricant would result in a comparatively greater friction power loss. The viscosity of the lubricant at the piston-ring cylinder liner interface varies during the cycle as the shear rate experienced by it changes [3].

The importance of studying cavitation lies in the fact that it can cause tremendous damage to fluid handling machinery. It is therefore, a matter of great significance to study the effect of oil properties and their subsequent influence on cavitation, whether it is a large cavitation area at the interface of the ring-liner assembly or cavity-shape details regarding the size (width or length) of the cavitation forms at oil film rupture.

\section{Experimental Set-up}

The study was conducted in two experimental test rigs. A single-ring test rig that simulates the piston-liner movement but the motion is inversed, i.e. it consists of a steady piston-ring section of $5 \mathrm{~mm}$ width that is fixed under a flat liner section. A single cylinder Lister-Petter PHW1 diesel engine was also used for motoring experiments. The liner as well as the cylinder block were modified to accommodate glass window sections and view for the visualization experiments [1].

\subsection{Single-Ring Test Rig}

The single-ring test rig itself is capable of providing valuable information about piston-ring lubrication under simplified conditions, by eliminating the in-cylinder complications resulting from ring dynamics, thermal and elastic deformation of the rings, starved lubrication, circumferential ring variations, lubricant degradation and blow-by. Easy access to the ring-liner interface is its basic characteristic and thus, a better understanding of the lubrication and the lubricant characteristics can be achieved through a series of measurements from sensors that are mounted in this experimental set-up.

Oil film thickness is measured both by a capacitance sensor (electrical method) and an LIF (laser induced fluorescence) sensor that is mounted within the liner body and reciprocates according to the testing conditions set. Friction is measured via a force measurement sensor mounted outside the oil bath. A second liner section was manufactured that incorporates an ultra-miniature pressure transducer providing valuable oil film pressure data that can be accompanied by visualization experiments that were carried out with another liner, specially manufactured for that reason. This liner which consists of a glass section enabled visualization of the oil film at the ring-liner interface that helped in studying further cavitation and extracting useful results, after the initial evidence of cavitation from the LIF measurements. In Figure 1a) the glass liner section is shown and in Figure 1b) the piston-ring section set-up for the experiments, the oil jets, the friction and the capacitance sensor.

The speed, load, temperature and stroke length can all be adjusted. Measurements of oil film thickness, oil film pressure across the ring and total axial friction can be recorded $[1,3]$. 


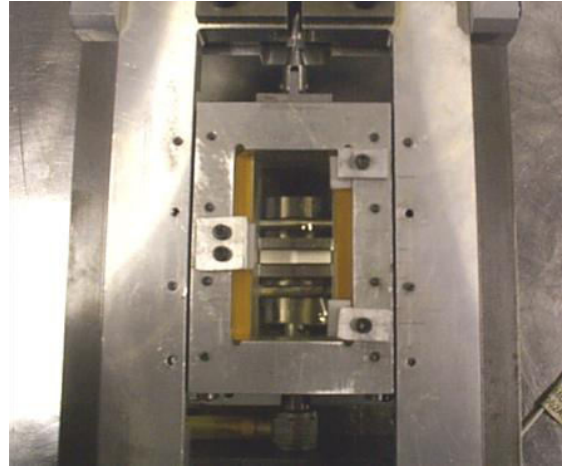

a)

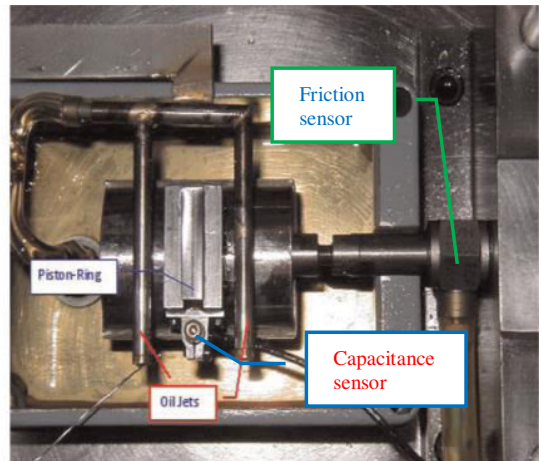

b)

Fig. 1. a) The glass liner section and b) Close-up of the piston-ring specimen

\subsubsection{Single-ring test rig results}

The evaluation of pressure measurements and visualization tests showed clear forms of cavitation at the interface between the piston-ring and the liner surface. The following schematic shows different forms of cavities that start appearing at the surface of the pistonring specimen and after developing in size as fern-shaped cavities after the dead centers of the stroke, eventually they turn into fissure shaped cavities and then string cavities that do not reach the trailing edge of the piston-ring. Close to mid-stroke where the liner reaches its maximum speed due to its sinusoidal movement, the string cavities reach the trailing edge of the piston-ring and the cavitation becomes open form instead of closed that was before. According to Figure 2 the stages of cavitation initiation and development at the beginning of the stroke can be seen:
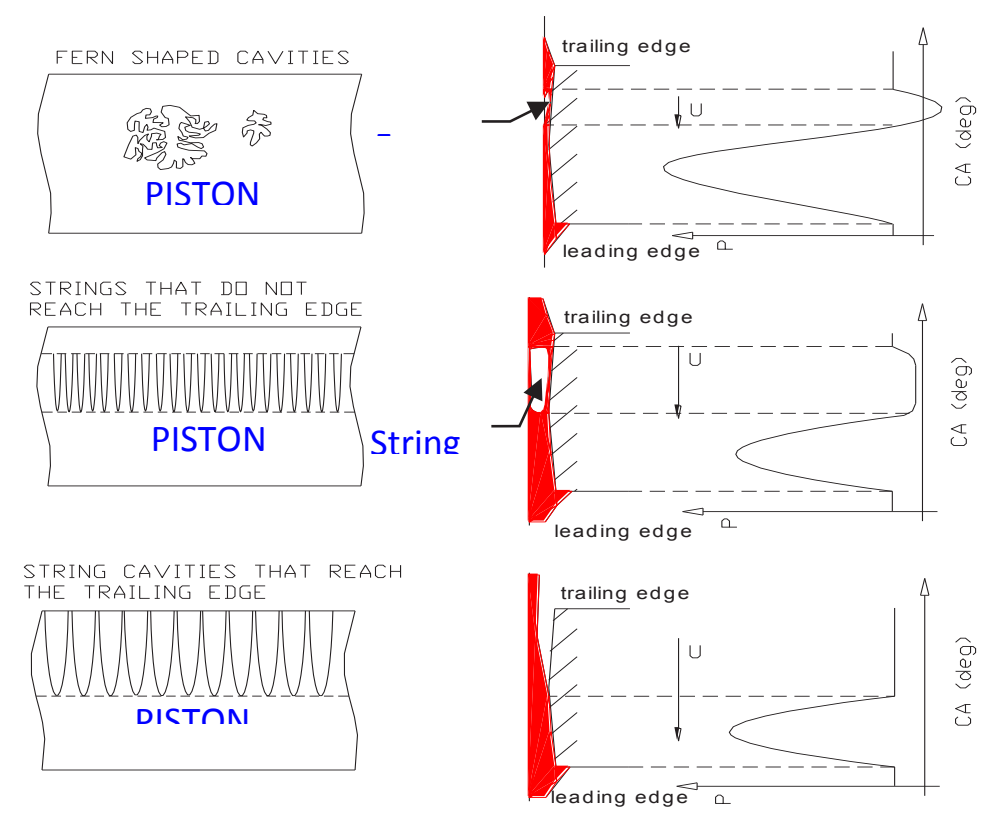

Fig. 2. Stages of cavitation initiation and development from the beginning of the stroke upto mid-stroke. The stages are accompanied by pressure measurements [4]. 
From the capacitance signal measurements shown in Figure 3, it was evidenced that there is a hump in the minimum oil film thickness measurement, which is happening close to mid-stroke and according to the accompanying figures from visualization experiments, it was verified that this hump is caused by cavitation stages transition (see Figure 3 b)) [4].

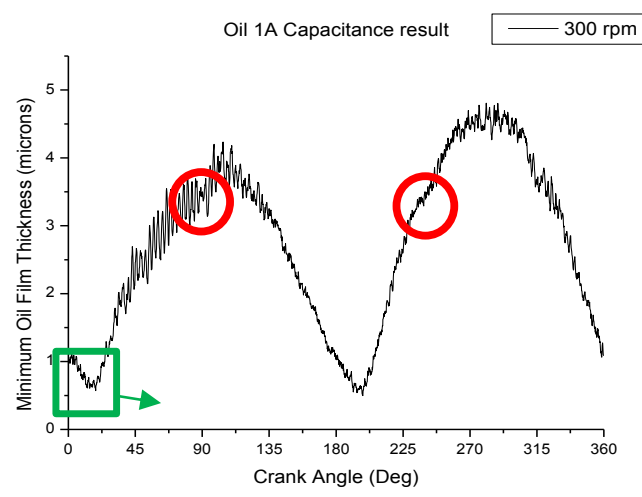

a)

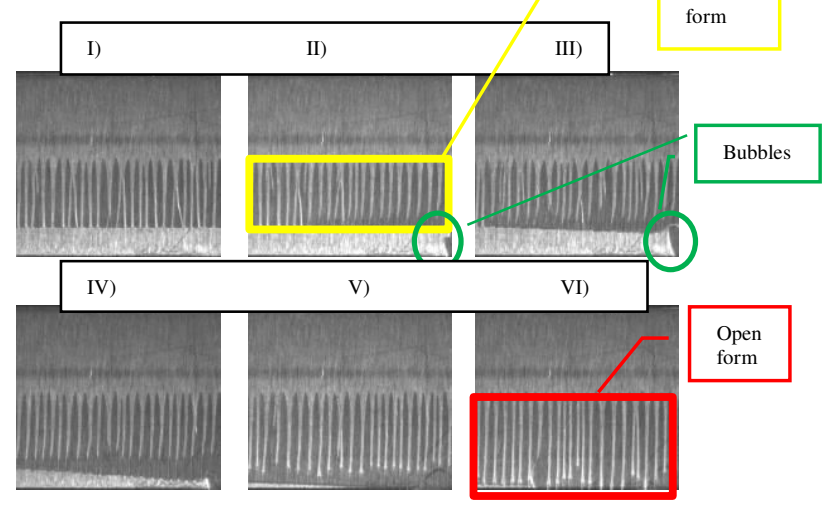

b)

Fig. 3. a) Minimum oil film thickness measurement from the capacitance sensor. Red highlighted areas show the cavitation conditions transition b) cavitation condition transition from closed form to open form and bubble suction are recorded in the visualization experiments [4].

When the cavitation strings do not reach the trailing edge of the ring, the pressure in the closed form cavity is sub-atmospheric (Figure 3b) I), II), III)). When the transition from this cavitating condition to the consequent open form happens, there is this change-hump in the minimum oil film thickness measurement as seen in the red highlighted areas of the capacitance signal (Figure 3a)) and eventually the negative oil film pressure transforms into atmospheric (Figure 3b) IV), V) and VI)).

What should be discussed further on is the eventual effect of cavitation on friction measurements. First, a friction signal is presented, so that a complete picture of the types of lubrication and its effects can be analysed. A typical friction signal is in Figure 4.

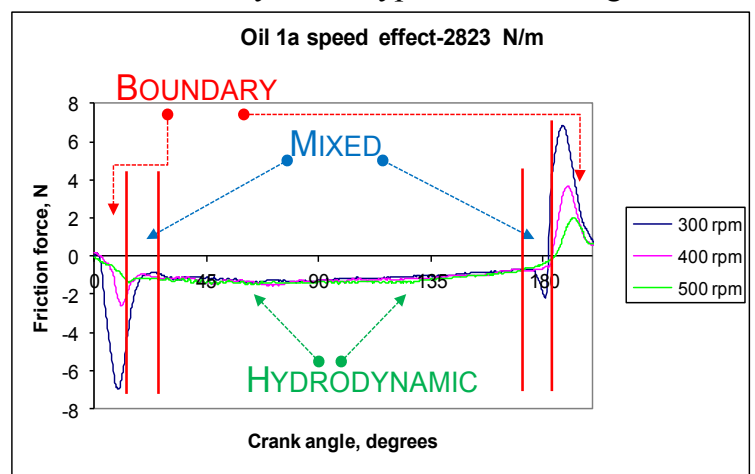

Fig. 4. Friction signals derived from high load test case for three different speeds

Different lubrication regimes are identified for the downstroke friction signal at high load for three different speeds. The friction force at contact (dead centers) is due to asperity interaction and the lubrication regime is boundary. Next to the boundary lubrication region, there is a mixed region where the frictional force due to asperity interaction is much larger than the viscous losses. This results in a point of minimum $\mathrm{C}_{\mathrm{f}}$ (friction coefficient) as the 
ring makes a transition from mixed to full film lubrication [5] and the hydrodynamic region where all frictional losses are due to viscous drag.

Extensive parametric study [3] showed that the friction spikes are dependent upon piston-ring curvature, speed, load and temperature conditions, and high temperature high shear (HTHS) viscosity of the lubricant. Power losses were calculated for the different test cases, for the whole stroke and for the boundary lubrication region so that data could be compared and useful results derived from the parametric study to be evaluated. Figure 5 shows the effect of high-temperature high shear viscosity on the friction peaks for different lubricants tested at BDC (bottom dead center).

Friction peaks for all oils tested at $300 \mathrm{rpm}$ -

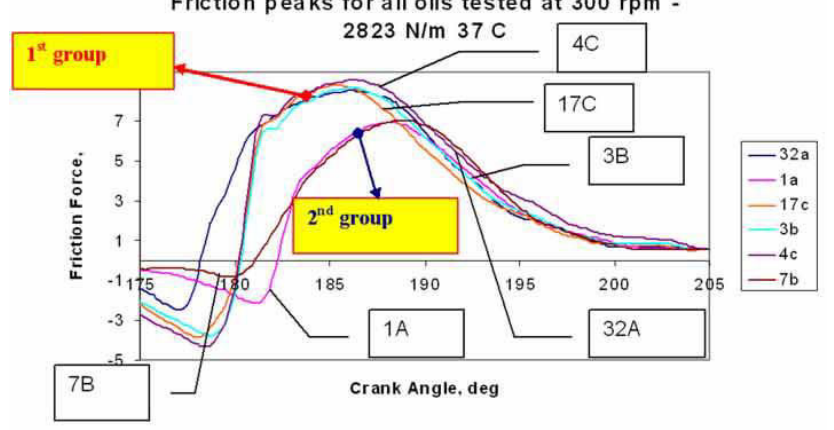

Fig. 5. Parametric friction force measurements at BDC for 6 different lubricants [3].

Table 1. Tested oil properties

\begin{tabular}{|c|c|c|c|}
\hline Blend Code & $\begin{array}{c}\text { Grade } \\
\text { (SAE) }\end{array}$ & $\begin{array}{c}\text { HTHS } \\
(\mathrm{mPas})\end{array}$ & V40 (cSt) \\
\hline \hline 1A & $5 \mathrm{~W} 40$ & 3.60 & 80.50 \\
\hline 7B & 0W30 & 3.64 & 66.82 \\
\hline 3B & 0W30 & 3.30 & 68.93 \\
\hline 17C & 0W30 & 3.39 & 67.78 \\
\hline 4C & 0W30 & 2.95 & 52.90 \\
\hline 32A & 0W30 & 3.35 & 65.97 \\
\hline
\end{tabular}

Each group of oils (oil properties in Table 1) in Figure 5 has similar high temperature high shear (HTHS) viscosity. Furthermore, in Figure 5 one can see the effect of viscosity on friction signals at the dead center of the stroke. This parametric study showed that at $33{ }^{\circ} \mathrm{C}$, the effect was that the oil with the lowest viscosity $\mathrm{V}_{40}$, oil $4 \mathrm{C}$, had the highest friction peak and the oil with the highest viscosity, 1A, had the lowest friction peak (at boundary lubrication conditions) [3].

For the same oil, high temperature testing showed high friction results for the boundary and mixed lubrication region, whereas in the hydrodynamic region, the results showed that there is no significant change in the friction force value. Capacitance measurement of minimum oil film thickness showed that oil film thickness gets lower as temperature rises.

\subsection{Single-Cylinder Diesel Lister Petter Engine}

A single cylinder Lister-Petter PHW1 engine that was used for visualization imaging in motoring tests was modified in the single cylinder engine block and liner to accommodate the experiments. The main purpose of these modifications was to capture the same type of images, as in the case of the single-ring test rig, and results concerning the initiation and development of cavitation to be extracted for the respective engine experiments. The visualized area, though, was not covering the whole of the stroke length and visualization 
with a CCD camera was depending on the lighting provided by a flash lamp with fibres to guide the light, depending always on the proximity of the camera to the engine block and the position of the camera along the stroke length $[1,6]$.

\subsubsection{Single-cylinder diesel engine test results}

The acquisition of images were not as clear as in the case of the single ring test rig, but similar forms of cavities were visualized and as a result, a hypothesis was made for the possible cavitation stages of the engine case, for the motoring tests. Possible cavitation stages were established as a result of the visualization imaging, combined with possible respective pressure measurements. Images of cavitation (string form) on the surface of the top compression piston-ring similar to the ones that were acquired in the imaging testing of the single-ring test rig were identified. Proper magnification enabled the visualization and analysis of these cavities $[1,6]$.

Images taken at $45 \mathrm{~mm}$ from TDC at a recording rate of 15000 frames per second showed oil accumulated on the second land travelling axially in the opposite piston direction and entering the converging wedge of the second compression ring. More testing showed that oil bubbles and droplets forming a ligament on the first piston land and travelling upwards to the top compression ring. This oil film is driven by inertia forces and depends on the volume of oil available on the piston land [7].

To capture the transient cavitation structures the recording rate was set to 30000 frames per second. The fern-shaped structures or the cavitation fissures and strings were not captured as opposed to the test rig. It is thought that engine cavitation structures, during their development are much smaller and short lived compared to the test rig and lubricant starvation should also be considered instead of the fully flooded lubrication conditions in the test rig experiments. It was, however, observed, that oil film enters the converging wedge of the piston-ring and later on in the stroke it exits the diverging wedge as bubbles. Then bubbles coalesce into an oil puddle growing in size and moving towards the first land. These results accompanied with sub-atmospheric pressure readings from engine testing suggest that the oil film cavitates and then disintegrates into bubbles. Oil droplets and mist can initiate a larger oil transport towards TDC [7].

\section{Discussion}

Quickly after the dead center, a new cavitating region forms at the trailing edge of the contact. Although these two cavitating regions only coexist for a brief period, together with a very low entrainment motion and high contact loads, lead to thinner films and higher friction forces as shown in the respective Figures.

This region which is unwetten by the lubricant and is exposed to outlet gas pressure reduces the required hydrodynamic load capacity from the lubricant film. At this stage, we see in the following schematic (Figure 6) the reduction or increase of the "effective" hydrodynamic film area for different operating conditions of speed and load. The higher the speed, the less support from the hydrodynamic film is provided. The higher the load, the greater hydrodynamic support exists at the piston-ring liner interface (for the hydrodynamic lubricant film condition).

Thus, there are extensive cavitating regions which contribute no radial support but which generate frictional losses. The cavitating region is different in size because of the geometry of the piston-rings, but it is not clear whether cavitation development at the beginning of the stroke plays a significant role in the measured friction peaks (boundary lubrication) and cavitation initiation with the fern-shaped or fissure shaped cavities [1]. 
The lubricant film thickness has a major effect on cavitation behaviour. Fluid properties play the primary role in the formation and collapse of cavities. Fluid properties influence cavitation behaviour in lubricants directly by introducing changes in the film thickness [8].

It is known that the piston-ring encounters the entire range of lubrication regimes through each stroke [5]. Thus, an investigation into frictional losses at the piston-ring cylinder liner contact must take into account the transitions from a state of full film lubrication to boundary lubrication.
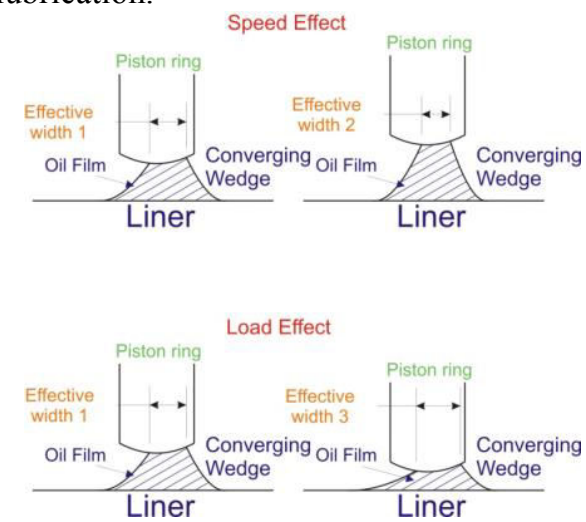

Fig. 6. Speed and load effect on the "effective" piston-ring width [1]

The "effective" piston-ring width, which is the corresponding piston - ring width for the oil film pressure curve obtained, is changing for each measurement. Figure 6 is providing an insight of the "effective" piston - ring width and how does it change with varying speed and load [1].

What should be noticed in the case of the parametric oil film pressure measurements for each piston - ring specimen, is that for the load variation tests the pressure peaks appear more uniform. Cavitation generation is largely dependent on pressure fluctuation.

The "effective" ring width is altered as it gets wider by a small margin while load increases, whereas the pressure peaks do not have a big variation for each testing condition i.e the curve peaks are slightly shifted between them; [1]. Initially, in the pressure testing results for different piston - rings, the slight variation of the mid-stroke setting of the liner relative to the ring was blamed for the pressure peaks shift that was noticed for the different specimens. Without this reason being excluded as it was possible to set the stroke length only relative to marks and a reference stroke measurement point, Figure 6 provides an additional explanation. It is important to note that the "effective" ring width is varied due to the different ring curvature and it is also necessary to stress the fact that the point where the oil film attaches to the piston-ring changes as well due to the operating conditions. So, for the same speed and load different pressure peaks crank angles are obtained for ring specimens with different curvature [1].

\subsection{Cavitation and Wear}

Cavitation damage results mainly from liquid impact on the bearing wall during cavity collapse. Since viscosity inhibits cavity growth and collapse, it is expected that the collapse velocity will be lower in a Newtonian lubricant than in a shear thinning lubricant. Shear thinning influences cavitation behaviour primarily by modifying the film thickness [8]. The shear thinning feature of multigrade lubricants and use of thinner monograde oils can 
generally reduce frictional losses but this may result in an increased wear of the cylinder liner.

The hydrodynamic pressure is strongly dependent on the thickness of oil film and the effective width of the ring surface. When the ring-liner tribological conjunction is under the starved lubrication condition, the oil attachment and detachment would be changed. The effect of film thickness on cavitation behaviour must also be taken into account while assessing the influence of viscosity and cavitation damage [8].

When the liner decelerates, the interface reaches a state of mixed lubrication and asperity interactions and frictional losses continue to decrease until the liner reaches boundary lubrication close to BDC as the squeeze film effect prevails [1].

Oil viscosity and oil film thickness are characteristics that affect friction losses. Higher viscosity oils provide adequate wear protection but movement creates more resistance and high frictional losses when measured for the total stroke length [3]. Lower viscosity oils reduce frictional losses and increase efficiency but high volatility leads to hydrocarbon emissions. The reduction of oil viscosity makes the lubrication around the dead center of the stroke much more severe [9]. It is, of course, a matter of great importance to further investigate lubricant properties and friction as there is always a trade off between performance and emissions control.

The size of the cavitation zone in shear thinning lubricants is significantly larger than that for Newtonian lubricants. This difference in cavitation behaviour arises as a result of the lower tensile strength of shear thinning fluids [8].

According to Kim et al [10], the striated oil film with fingerlike cavities is observed and verified and friction force in the region of oil film rupture must be taken into account.

A series of additives can be used to reduce friction under boundary lubricating conditions. Surface active agents are closely related to extreme pressure additives in structure and mode of action (boundary lubrication). Viscosity index improvers hold the decline in oil viscosity with temperature. Multigrade oils allow an acceptable engine operation over a wide temperature range.

The problem of changing the friction properties of the ring-liner interface was addressed with the introduction of the textured liner. Surface texture changes the friction properties at the boundary-mixed lubrication region. As texture features work as lubricant microreservoirs, the oil feeding induced by the features should also be considered as the additional source of oil. The grooves that contribute to the surface texture can be in either side, (ring or liner) and studies showed the existence of microcavitation on these grooves.

\section{Conclusions}

Cavitation causes the effective load area to shrink and the load capacity declines, even if specific hydrodynamic pressures remain high. The cavitating region for the different lubricants and piston-rings should be considered. Friction peaks appear due to the collapse of the squeeze film close to the dead centres of the stroke, they are affected by speed, load, viscosity and high temperature high shear viscosity and provide indications of wear development at the piston assembly interface. Liner or ring etching changes the lubrication regime at the areas of interest.

The lubricant film thickness has a major influence on the cavitation behavior, which in turn, affects the shape of the hydrodynamic pressure profile in the entrainment direction and consequently the ring-pack's load carrying capacity.

Extensive parametric lubricant study is required to combine the effect of cavitation initiation for different lubricants and the appearance of friction peaks combined with the calculation of total friction losses. 


\section{References}

1. P. Dellis, Aspects of lubrication in piston cylinder assemblies, (PhD Thesis, Mech. Eng. Dept., Imperial College London, 2005).

2. M. Priest, D. Dowson, C. M. Taylor, Proc. Instn. Mech. Engrs., Part C:J. Mech. Eng. Sc., 214(C3), 435-447, (2000).

3. P. Dellis, Proc IMechE, Part J: J Engin. Trib.; 224(5): 411-426, (2010).

4. P. Dellis, and C. Arcoumanis, Proc. IMechE, Part J: J. Engin. Trib., 218, 157-171. DOI: 10.1243/1350650041323340, (2004).

5. N. W. Bolander, B. D. Steenwyk, F. Sadeghi, and G. R. Gerber, Proc. IMechE, Part J: J. Engin. Trib., 219(1), 19-31. DOI: 10.1243/135065005X9664 (2005).

6. P. Dellis, Tribology in Industry, 39 No. 2, 260-269, DOI: 10.24874/ti.2017.39.02.14, (2017).

7. A. Dhunput, Oil transport in piston ring assemblies, (PhD Thesis, School of Eng. And Math. Sc., City University London, 2009).

8. A. Rastogi, and R. K. Gupta, SAE paper 922286, (1986).

9. Y. Wakuri, M. Soejima, Y. Ejima, SAE paper 952471, (1995).

10. S. Kim, A. Azetsu, M. Yamauchi and T. Someya. JSME Int. J. Ser. C, 38(4), 783789, (1995). 